PAPER

\section{Off-axis compensation of attosecond pulse chirp}

To cite this article: C Hernández-García and L Plaja 2012 J. Phys. B: At. Mol. Opt. Phys. 45074021

View the article online for updates and enhancements.

\section{Related content} Tailoring isolated attosecond pulses using Carlos Hernández-García and Luis Plaja

Group velocity matching in high-order harmonic generation driven by midinfrared lasers

C Hernández-García, T Popmintchev, M M Murnane et al.

Spectral splitting and quantum path study of high-harmonic generation from a semiinfinite gas cell

W Cao, G Laurent, Cheng Jin et al.

\section{Recent citations}

- Transverse phase matching of high-order
$\frac{\text { harmonic generation in single-laver }}{\text { graphene }}$
Roberto Boyero-García et al
- Conservation of Torus-knot Angular
$\frac{\text { Momentum in High-order Harmonic }}{\text { Generation }}$
Emilio Pisanty et al
- Annie Klisnick et al

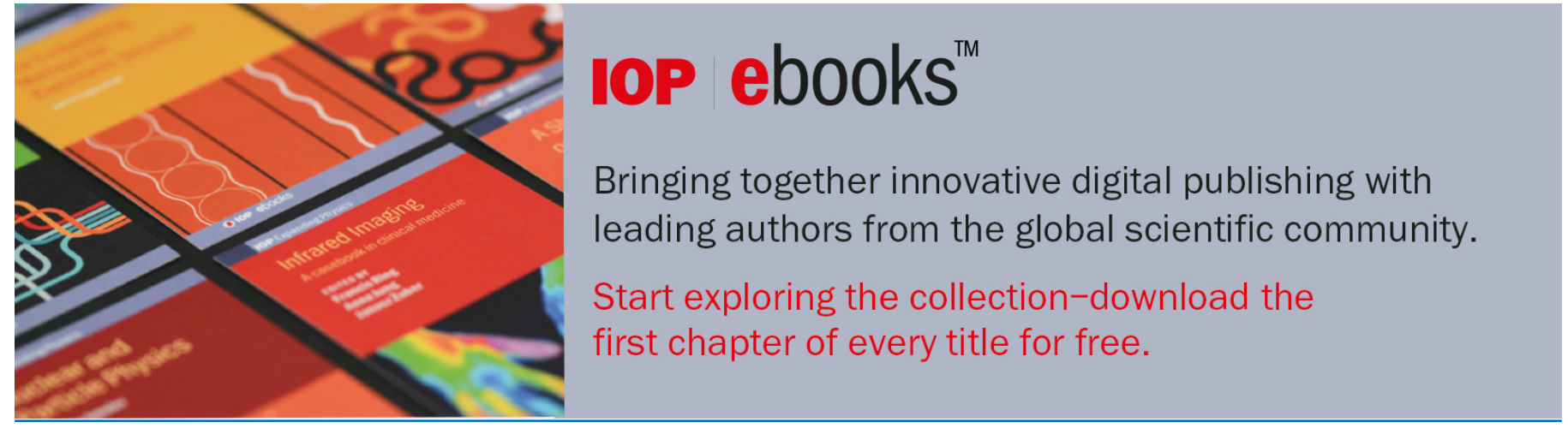

This content was downloaded from IP address 212.128 .135 .41 on $28 / 05 / 2021$ at $14: 48$ 


\title{
Off-axis compensation of attosecond pulse chirp
}

\author{
C Hernández-García and L Plaja \\ Grupo de Investigación en Óptica Extrema, Universidad de Salamanca E-37008, Spain \\ E-mail: carloshergar@usal.es
}

Received 15 September 2011, in final form 13 December 2011

Published 16 March 2012

Online at stacks.iop.org/JPhysB/45/074021

\begin{abstract}
By performing calculations on the generation of attosecond pulses by intense laser fields, we demonstrate that the pulse temporal width is sensitive to the angle of emission. We show that this effect results from the interference of the short and long electronic trajectory contributions, responsible for high-order harmonic generation. In particular, we find that the shortest pulses are emitted off-axis, which are substantially shorter than those emitted on-axis, where single trajectory contributions dominate.
\end{abstract}

(Some figures may appear in colour only in the online journal)

\section{Introduction}

One of the most exciting perspectives of high-order harmonic generation by intense lasers is the possibility of synthesizing XUV pulses of sub-femtosecond duration [1-3]. Attosecond pulses are obtained by the selection of the higher frequency part of the harmonic spectrum which conform the plateau region. For the correct synthesis, the spectrum should approach two conditions: on one side its structure should approach that of a frequency comb, in which the harmonic intensities are similar; on the other side the relative phase between the harmonics should be nearly constant (phase locking) [4]. Fortunately, these two conditions are approached in the typical harmonic spectra generated during the interaction of intense fields with matter. Nevertheless, the macroscopic conditions of the experiment play an important role for the final phase locking of different harmonics [5, 6].

According to the three-step model [7, 8], the generation of the most energetic harmonics by intense fields can be understood with simple semiclassical arguments. The energetic photons are generated during the rescattering of ionized electrons with the ion. Therefore, in the first step, the atomic electron should tunnel ionize through the potential barrier formed by the Coulomb potential, shaped by the electric field. After the release, in the second step, the electron is accelerated by the electric field and driven back towards the parent ion. The high-frequency radiation is emitted in the last step, during the electron rescattering with the parent ion.

The particular properties of the harmonic radiation, and therefore of the synthesized attosecond pulses, can be well understood in semiclassical terms. Typically, in each halfcycle there are two possible electron trajectories leading to the same kinetic energy at recollision, and therefore two possible semiclassical paths for the generation of the same harmonic (each named according to the excursion time as short and long trajectories). According to the Feynman description, each semiclassical trajectory has associated a phase corresponding with the mechanical action. Since this phase is translated to the harmonic radiation, the final harmonic emission reflects the interference between the contributions of the short and long trajectories [9].

Attosecond XUV pulses can be synthesized by selecting a window of the higher harmonic spectrum, using an appropriate filter. Physically, this corresponds to the isolation of the burst of radiation associated with the most energetic rescattering events, having typical durations well below the laser cycle. As different kinetic energies scatter at slightly different times, the attosecond burst is chirped and, therefore, its temporal duration is not the Fourier limit [10-12].

On the other hand, for harmonic generation below the cutoff, short and long trajectories do not rescatter simultaneously. Consequently, selecting only the contribution of a single trajectory, one can optimize the width of the attosecond pulses [13-15]. In this paper we shall show that, for particular angles of detection where both the short and long trajectories are relevant, the destructive interference of these contributions can lead to a final attosecond burst with smaller duration than that corresponding to a single trajectory. Spectrally, this corresponds to the optimal compensation of the chirp of the attosecond pulse. 

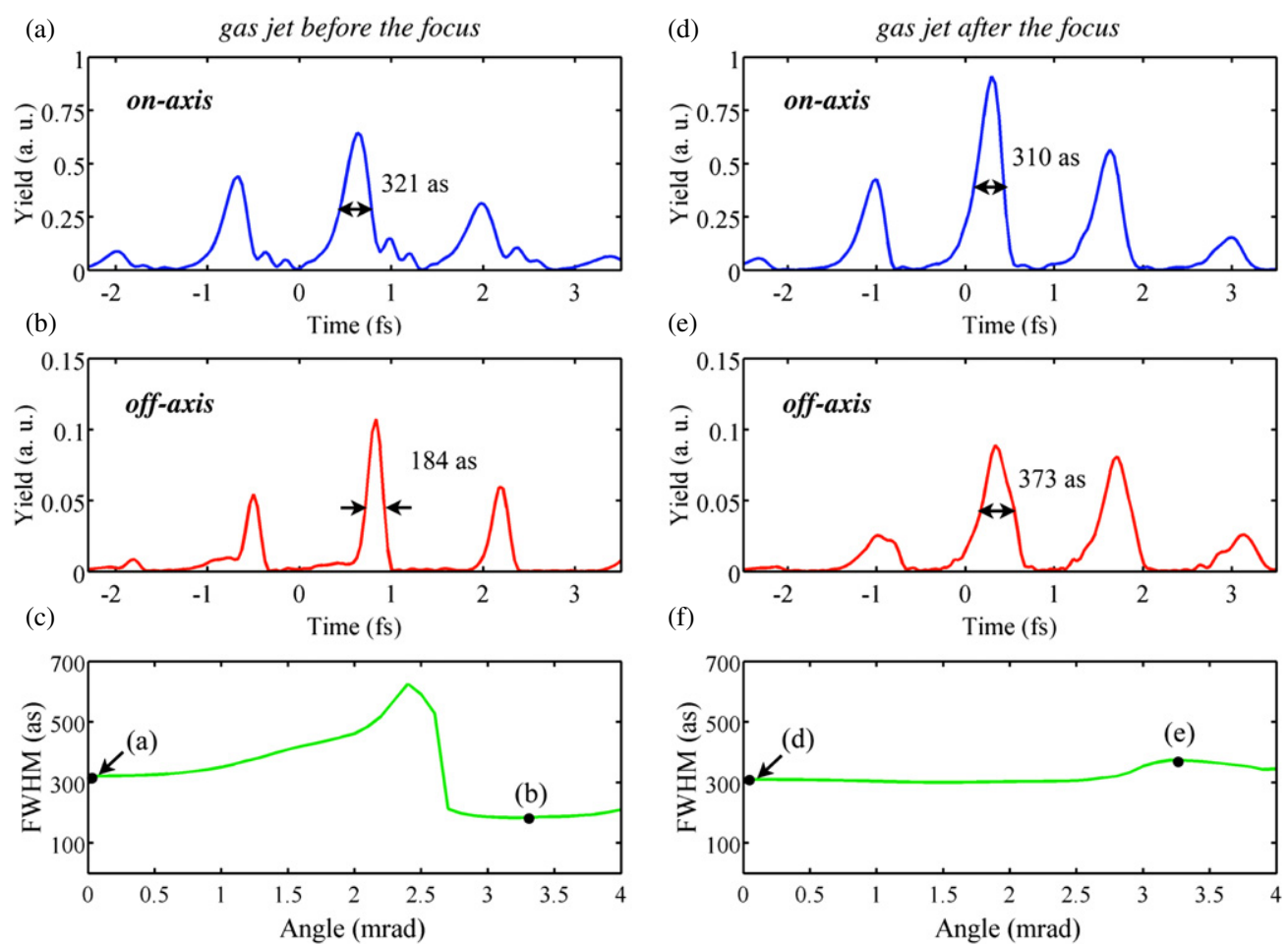

Figure 1. Temporal distribution of the attosecond train of pulses when the high-order harmonics are detected in a window (a) on-axis and (b) centred at $3.3 \mathrm{mrad}$, when the gas jet is placed $1 \mathrm{~mm}$ before the focus position. (d) and (e) represent the same situation as (a) and (b), but when the gas jet is placed $1 \mathrm{~mm}$ after the focus position. (c) and (f) show the FWHM of the pulses synthesized as a function of the detection angle, when the gas jet is located $1 \mathrm{~mm}$ before and after the focus, respectively.

\section{Methods}

We perform our computations assuming an 8 cycle $(7.5 \mathrm{fs}$ FWHM) $\sin ^{2}, 800 \mathrm{~nm}$ fundamental field with intensity at focus $\simeq 2.45 \times 10^{14} \mathrm{~W} \mathrm{~cm}^{-2}$ and polarized along the $x$ direction. The beam is assumed Gaussian (propagating along the $z$ direction) and taken directly as an input to our calculations. The beam waist at focus is $22.5 \mu \mathrm{m}$ and the confocal parameter $b=3.98 \mathrm{~mm}$. The target is modelled as an argon gas jet flowing perpendicular to the Gaussian beam. The gas jet profile is constant in the $x$ direction, while Gaussian in the $y$ and $z$ directions, with the FWHM of $500 \mu \mathrm{m}$ and peak density of $10^{18}$ atoms $\mathrm{cm}^{-3}$. For the angular resolved detection, we consider a window of $0.5 \mathrm{mrad}$ in the far-field profile that will correspond experimentally to an annular gate.

Our propagation code [16] is based on the discrete dipole approximation and includes the effect of free electrons. The use of discrete dipoles allows us to compute the harmonic field at the detector directly as the summation of the fields emitted by the atomic dipoles at different points in the target. The kernel of our propagation code is the single-atom harmonic radiator solver that computes the harmonic radiation from a single atom for the particular form of the electromagnetic field corresponding to the atom's position. Our single radiator solver is based on the SFA+ approach [17], an extension of the conventional strong field approximation approaches. The SFA+ approach includes part of the field dressing of the ground state and gives an accurate quantitative description of the harmonic spectrum, in agreement with the exact integration of the 3D time-dependent Schrödinger equation [18, 19]. A particular feature of the SFA+ is that it computes the harmonic emission from the full quantum approach, i.e. not resorting to the saddle point approximation. Therefore, in this paper, the concepts derived from the semiclassical approach are used for discussions but not in the actual computations. We have performed our calculations in Ar, using the Roothaan-HartreeFock wavefunction [20] for the ionization and rescattering matrix elements. The acceleration operator for hydrogen in [17] is replaced accordingly with the gradient of the Coulomb potential of the argon ion [21]. The attosecond pulses have been computed by the Fourier transformation of the harmonic spectrum detected at a particular angle, after filtering out of the low-energy part. We have simulated filtering with an aluminium plate by multiplying the total harmonic spectrum times the transmission function for this metal.

\section{Results}

We plot in figure 1 the train of attosecond pulses resulting from the Fourier transform of the harmonic spectra obtained with the numerical simulation explained in the preceding section. We have considered two different situations: target placed before the laser focus (left column: figures 1(a)-(c)) and after the focus (right column: figures 1(d)-(f)). It is already known $[22,23]$ that when the target is positioned after the focus, phase matching favours only short trajectories, becoming dominant at all detection angles. In contrast, when the target is located before the focus, both trajectories are present, with the short ones being dominant on-axis, whereas as the angle of detection increases, the contribution 

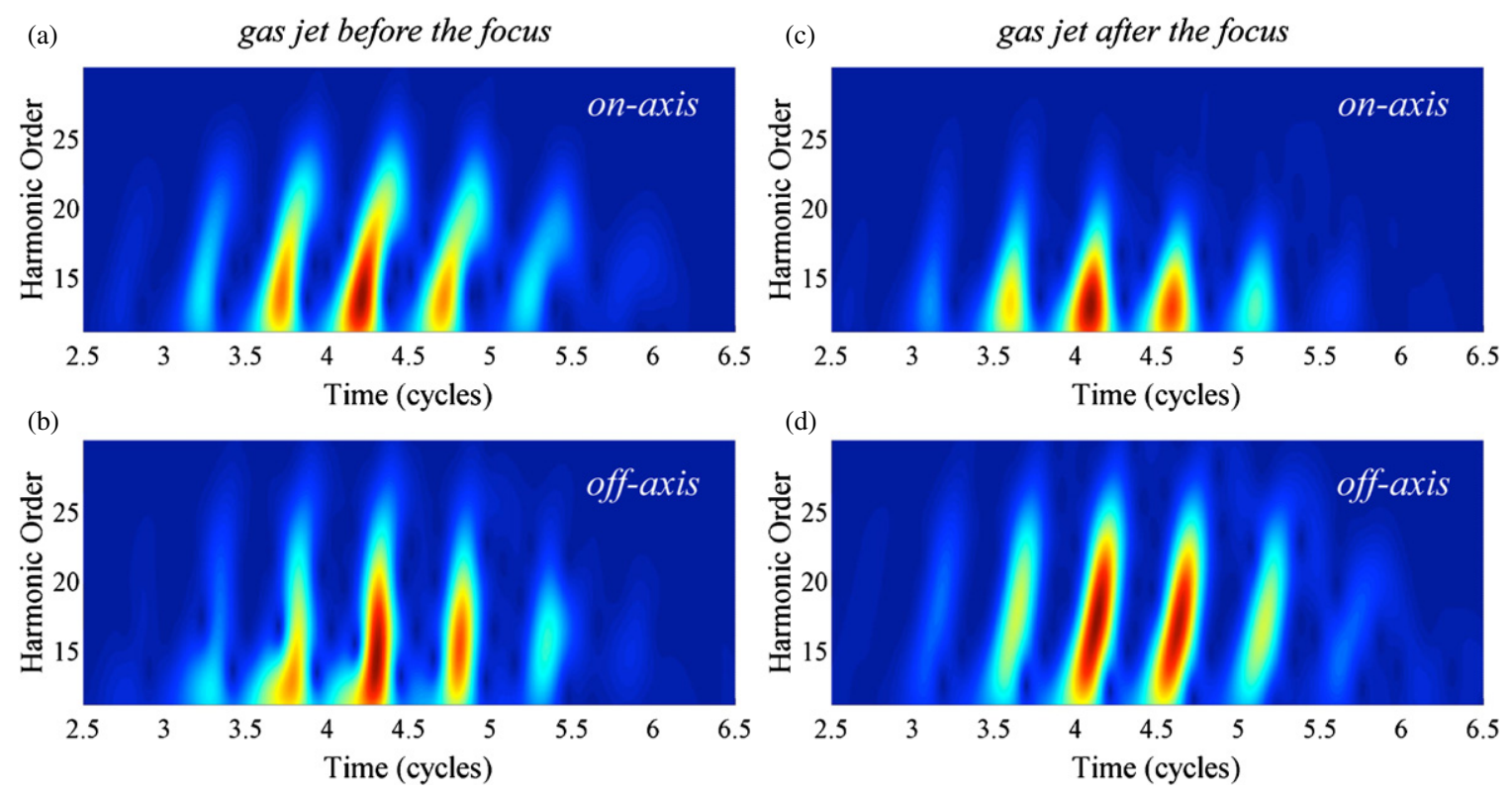

Figure 2. Wavelet analysis of the filtered harmonics of the spectrum, when they are detected in a window on-axis (first row) and at $3.3 \mathrm{mrad}$ (second row). From left to right, the gas jet is placed $1 \mathrm{~mm}$ before/after the focus position. The intensity is normalized in each graph. The temporal axis is in units of the laser cycle.

of long trajectories becomes gradually important. Therefore, when the target is positioned before the focus, there is a range of detection angles in which both trajectories are comparable. The first row in figure 1 (plots (a) and (d)) corresponds to the attosecond pulses detected on-axis, while the second row shows the off-axis detection (plots (b) and (e)), where the spatial window was centered at $3.3 \mathrm{mrad}$. Finally, the third row (plots (c) and (f)) shows the width of the most prominent attosecond pulse as a function of the angle detection. The main conclusion of this paper can be drawn by the inspection of plots (a) and (b), comparing the attosecond pulse obtained on- and off-axes. The reduction of the pulse width becomes apparent for the off-axis case, obtaining 184 as pulses in contrast with 321 as on-axis. To our understanding, this reduction has a physical explanation in the interference between the radiation bursts associated with the rescattering of the short and long paths. This interference is the most acute off-axis, where both trajectories have comparable weights, rather than that on-axis, where short trajectories dominate. As a further check of this idea, we should expect, in a situation where phase matching favours only short paths at any angle, no reduction of the attosecond width with the angle of detection, as no other contribution cancels the positive chirp due to the short trajectories. This situation is found when the target is located after the laser focus position, and the corresponding results are shown in the plots in the right column of figure 1. Plots (d) and (e) (target after the focus) show little variation in contrast with the cases (a) and (b) (target before the focus). Figure 1(f) shows that the attosecond pulse width remains practically insensitive to the angle of detection.

Consistently with our interpretation, we have also found narrower X-ray pulses for fundamental fields leading to isolated attosecond bursts, therefore excluding the influence of multiple rescatterings in the pulse off-axis narrowing.
A further insight into this process can be gained performing a wavelet analysis of the filtered spectrum at the detection angles depicted in figure 1 . The wavelet analysis is performed using a frequency window of length $3.5 \omega_{0}$, with $\omega_{0}$ being the frequency of the fundamental field. In the left column of figure 2, we show the time-resolved emission of the high harmonics (a) on-axis and (b) at $3.3 \mathrm{mrad}$, when the gas jet is placed before the laser focus. In the right column, the same angles are used but with the target after the focus. From the discussion above, cases (a), (c) and (d) correspond to situations in which the short trajectory dominates. Accordingly, the electron's recollision energy shows a roughly linear dependence on time, in which the lower harmonics are emitted before the higher ones, giving rise to a positive chirp typical from the harmonic spectrum emitted by short trajectories. Plot in figure 2(b) corresponds to the situation of figure 1(b), off-axis detection leading to the shorter attosecond pulses. In this case, the interference between paths results in a modulation of the emission time of successive harmonics, producing vertical wavelet structures. Thus, destructive interference tends to confine the harmonic radiation into a narrower window, reducing drastically the positive chirp associated with short trajectories alone.

In order to understand when the interference between short and long trajectories takes place, we have plotted in figure 3 the classical temporal dependence of the electron's recollision energy for a constant envelope incident field. We have highlighted a temporal region of about 0.15 laser periods (400 as) that includes the rescattering times where short (positive slope) and long (negative slope) trajectories overlap for the energy range corresponding to harmonics from 11 to 21. This demonstrates the existence of an overlap between both contributions during the temporal width of the attosecond pulse generated by a single trajectory (figures 1 (a) and (d)). Note that this interference occurs between the short and long trajectories 


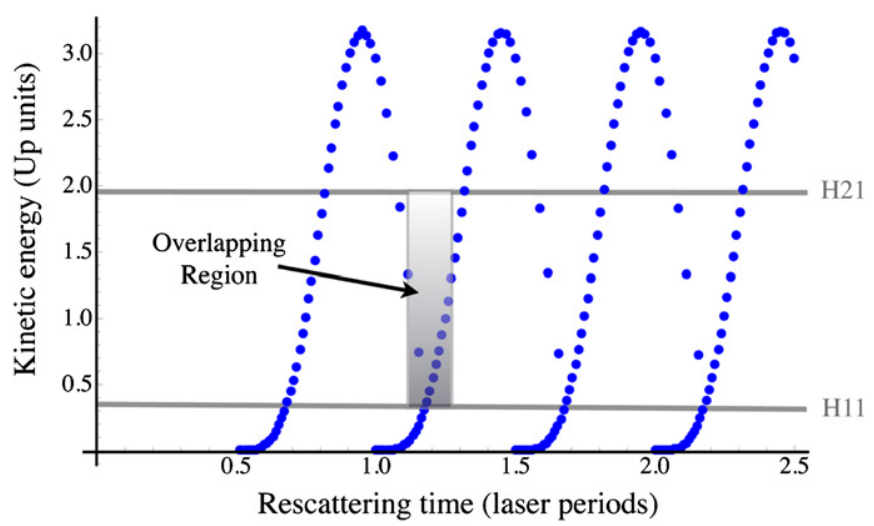

Figure 3. Classical temporal dependence of the electron's recollision energy for a constant envelope incident field. We have highlighted the time/energy region where long (negative slope) and short (positive slope) trajectories overlap for the energy range corresponding to harmonics from 11 to 21 . Long trajectories originated near a particular ionization maximum overlap with the short trajectories ionized at the following maximum.
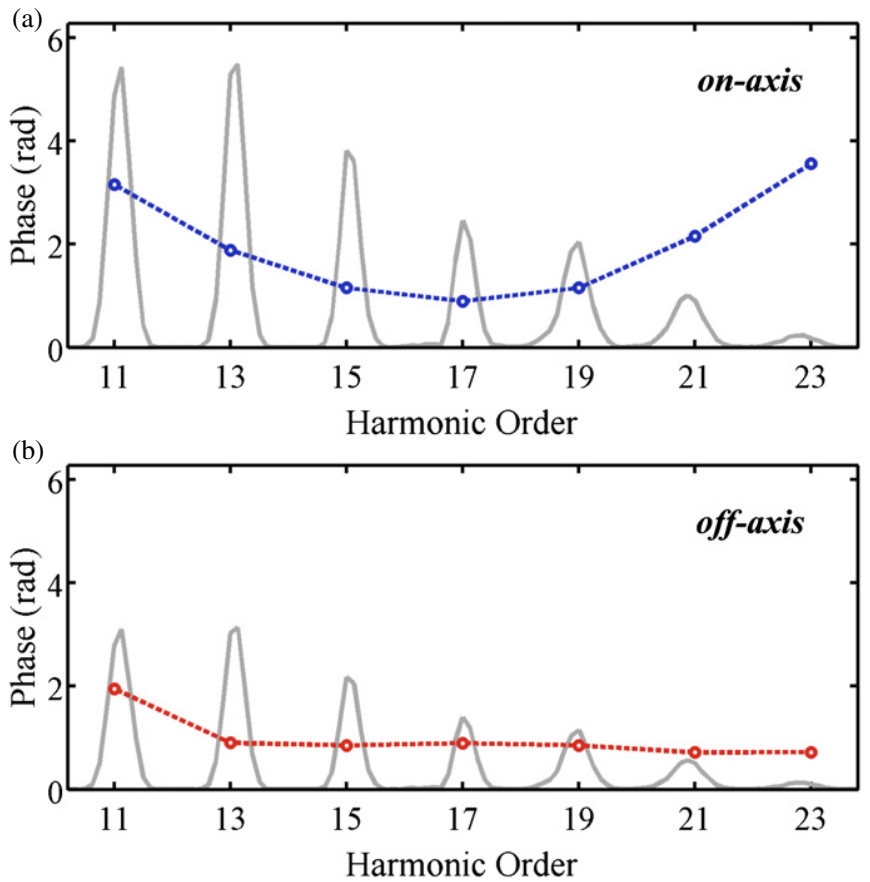

Figure 4. Quadratic term of the harmonic phase of the attosecond pulses when they are detected on-axis (a) and at $3.3 \mathrm{mrad}(\mathrm{b})$. At the background of both plots, we show the harmonic spectra.

originated, respectively, at two consecutive ionization maxima. Consistently, the modulations of the wavelet structures in figure 2(b) also appear at that highlighted region in figure 3.

In figures 4(a) and (b), we show the quadratic term of the spectral phase of the attosecond pulses showed in figures 1(a) and (b), respectively. When a spatial filter is placed on-axis to select the short trajectories, a positive chirp is present, in agreement with what was discussed before. In contrast, when filtering angularly the signal at $3.3 \mathrm{mrad}$ (figure 4(b)) the superposition of the contributions of both trajectories results in a reduction of the spectral chirp, and then in the phase locking of different harmonics.

\section{Conclusion}

We have presented theoretical results demonstrating the sensitivity of the attosecond pulse generation to the angle of detection. Our computations suggest that this sensitivity is a consequence of the interference of the two main contributions to the harmonic spectra, corresponding to short and long electronic trajectories. At some off-axis angles this interference results in the generation of attosecond pulses with durations well below those emitted on-axis, where only the short trajectories are relevant. We believe that this is a phenomenon potentially useful to reduce the attosecond chirping and, thus, to obtain shorter pulses in the laboratory.

\section{Acknowledges}

We acknowledge support from Spanish Ministerio de Ciencia e Innovación through the Consolider Program SAUUL (CSD2007-00013) and Research project FIS200909522, from Junta de Castilla y León through the Program for Groups of Excellence (GR27) and grant agreement ORDEN EDU/1708/2008, and from the EC's Seventh Framework Programme (LASERLAB-EUROPE, grant agreement 228334). LP acknowledges UK EPSRC-CAF grant EP/J002348/1 for supporting this research. We also acknowledge support from the Centro de Láseres Pulsados, CLPU, Salamanca, Spain.

\section{References}

[1] Paul P M et al 2001 Science 2921689

[2] Kienberger R et al 2004 Nature 427817

[3] Popmintchev T et al 2010 Nature Photon. 4 822-32

[4] Farkas G and Toth C 1992 Phys. Rev. A 168 447-50

[5] Gaarde M B and Schafer K J 2002 Phys. Rev. Lett. 89213901

[6] Ruchon T et al 2008 New J. Phys. 10025027

[7] Schafer K J et al 1993 Phys. Rev. Lett. 701599

[8] Corkum P B 1993 Phys. Rev. Lett. 711994

[9] Zair A et al 2008 Phys. Rev. Lett. 100143902

[10] Mairesse Y et al 2003 Science 3021540

[11] Varju K et al 2005 J. Mod. Opt. 52379

[12] Goulielmakis E et al 2008 Science 320 1614-7

[13] Mairesse Y et al 2004 Phys. Rev. Lett. 93163901

[14] Merdji H et al 2006 Phys. Rev. A 74043804

[15] Jin C et al 2011 Phys. Rev. A 84043411

[16] Hernández-García C et al 2010 Phys. Rev. A 82033432

[17] Pérez-Hernández J A et al 2009 Opt. Express 179891

[18] Pérez-Hernández J A et al 2009 Laser Phys. 19 1581-5

[19] Pérez-Hernández J A et al 2010 Laser Phys. 20 1044-50

[20] Clementi E and Roetti C 1974 At. Data Nucl. Data Tables 14177

[21] Tong X and Lin C 2005 J. Phys. B: At. Mol. Opt. Phys 382593

[22] Antoine A et al 1996 Phys. Rev. Lett. 771234

[23] Salières P et al 2001 Science 292902 